Yu.M. Khoverko, N.O. Shcherban

\title{
Electrical Conductivity and Magnetoresistance of Silicon Microstructures in the Vicinity to Metal-Insulator Transition
}

\author{
National University "Lviv Polytechnic", Lviv, st. Bandery str., 12, 79013 khoverko@lp.edu.ua
}

\begin{abstract}
Complex research of silicon microcrystals with specific resistance from $\rho_{300 \mathrm{~K}}=0.025 \mathrm{Ohm} \times \mathrm{cm}$ to $\rho_{300 \mathrm{~K}}=$ $0.007 \mathrm{Ohm} \times \mathrm{cm}$ doped with boron transport impurity to concentrations corresponding to the transition of metaldielectric and modified transition metal nickel at low temperatures to the temperature of liquefied helium $\mathrm{T}=4.2 \mathrm{~K}$ in magnetic fields up to $14 \mathrm{Tl}$. The features of electrophysical characteristics of samples at low temperatures in strong magnetic fields up to $14 \mathrm{Tl}$ are determined due to the influence of a magnetic impurity in semiconductor-diluted magnetism and the use of such crystals in sensors of physical quantities (temperature, magnetic field, deformation) is proposed.
\end{abstract}

Key words: jump conductivity; microcrystal; negative magnetic resistor; spin; cryogenic temperatures.

Article acted received 29.08.2018; accepted for publication 15.09.2018.

\section{Introduction}

The study of low-temperature characteristics of doped semiconductor microcrystals with impurity concentration in the vicinity of the metal-dielectric transition (MIT) has been sufficiently studied today [1,2], since they can provide information on the kinetic effects of these materials that can be used to create highsensitivity physical sensors capable of low temperatures and other basic elements of solid-state electronics. However, the study of the influence of external factors on the electrophysical properties of classical semiconductors such as silicon, germanium in view of the rapid development of modern magnelectronics (spintronics) is not well developed [3,4]. While in the spintronic many achievements were made on the basis of the study of the properties of metals [5], semiconductorbased spintronics is still under development. [6]. As is known $[7,8]$, the possibility of combining information about charged particles and spin degrees of freedom, semiconductors diluted with magnets - promising new materials in the field of spintronics $[9,10]$. In addition, it is known [11] that with the decrease in temperature, when ionization processes become impossible, charge carriers in the conduction band are freeze, their concentration becomes so small that the dominant contribution to current transfer begins to make jumps of electrons directly from donors (or acceptors) that occurs during account of a small but finite overlap of the wave functions of adjacent impurities. Such process of current surges by charge carriers between localized states characterized by hopping conduction. Numerous experimental facts and theoretical evidence convincingly prove that in the weakly doped and compensated semiconductor samples at rather low temperatures, the hopping mechanism of conductivity among others is the only mechanism that provides the transport of electrons $[12,13]$. Moreover the main characteristics of hopping transport determined by the structure of the impurity band, which, in turn, is defined as the properties of individual impurity centers and law electrostatic interaction between electrons and ionized impurity centers. Therefore, the introduction of a magnetic impurity into a crystal that is capable of polarizing charge carriers around itself makes it possible to create conditions in which it is necessary to take into account the polarization component for prediction of the magnetoresistance of crystals caused by the nature of the jump conductivity at low temperatures. In its turn, in the filamentous crystals, there were interesting magnetic properties associated with the possible formation of superparamagnetic clusters in crystals of submicron diameter [14]. In this regard, the study of magnetoresistance of silicon microcrystals, modified by an admixture of transition metals from a practical point of view is interesting primarily because the magnetic response of this material determines the possibility of microcrystals in sensors of physical quantities under the influence of an external magnetic field [15]. Besides, such studies provide an opportunity to deepen knowledge of the magnetosphere and the electrical conductivity of 
crystals [16], their behavior in different conditions, the nature and interconnection of these effects, etc. Finally, in the microcrystals there is a dimensional dependence of the magnetic susceptibility [17], which distinguishes them from bulk samples.

Thus, the purpose of the paper is to study the effect of a magnetic impurity on the movement of charge carriers in microcrystals doped with boron transport impurities to concentrations corresponding to the MIT at low temperatures to a temperature of liquefied helium $\mathrm{T}$ $=4.2 \mathrm{~K}$ in magnetic fields up to $14 \mathrm{~T}$ for the development of sensors physical quantities, able to work under harsh conditions.

\section{Details of the experiment}

The object of the research was microcrystalline silicon p-type conductivity, grown by the method of chemical gas transport reactions in the form of filamentous crystals in length $2 \div 5 \mathrm{~mm}$ and diametr $20 \div 60 \mu \mathrm{m}$ with crystallographic orientation $\langle 111\rangle$. To obtain p-type conductivity crystals in the process of growth drowned with boron impurities, loading boron anhydride into the ampoule $\left(\mathrm{B}_{2} \mathrm{O}_{3}\right)$, and gold and platinum, as initiators growth of whiskers. The concentration of charge carriers in crystals was in the range of $2 \times 10^{18}$ to $2 \times 10^{19} \mathrm{~cm}^{-3}$, covering the concentration range $\left(5 \times 10^{18} \mathrm{~cm}^{-3}\right)$ in the vicinity of the MIT. The nickel film was applied by the method of electrolytic chemical deposition, and further annealing at $\mathrm{T}=580{ }^{\circ} \mathrm{C}$ by means of low temperature diffusion led to the creation of profiled impurity zones, which, on the one hand, allowed the formation of ohmic contacts to the thickness of the crystal, and, on the other, the zones that could polarize the surrounding impurities in the thicker of the crystal. Investigation of conductivity behavior of deformed and non-deformed samples, magnetoresistance (MR) micro and nanostructures of silicon at temperatures $4,2-300 \mathrm{~K}$ in high magnetic fields up to $14 \mathrm{~T}$ conducted at the Institute of Low Temperatures and Structural Research (Wroclaw, Poland). Samples were placed in a special insert and placed in a helium cryostat where it happened cooled to a temperature of $4.2 \mathrm{~K}$ to room temperature sample was heated with a special insert from a bifilar heater wire wound on the body insert.

Stabilized electric current $1-100 \mu \mathrm{A}$ in the measuring circuit the source of the current was determined Keithley-224 depending on the resistance of the sample to be studied. The electrical voltage at the samples contacts, the output signal of the thermocouple and the magnetic field sensor were measured with digital voltmeter Keithley-199 and Keithley-2000 with accuracy up to $1 \mu \mathrm{V}$ with the simultaneous automatic registration of impressions through a parallel port of the personal computer and their visualization on the monitor screen and the recording of an array of data in the files.

To study the properties of silicon micro and nanostructures in magnetic fields up to $14 \mathrm{~T}$, a special method was used that allows to measure magnetoresistance at different fixed temperatures in the range from 4.2 to $300 \mathrm{~K}$. Measurement of field dependencies of MR was carried out in the range of magnetic fields $0-14 \mathrm{~T}$ for fixed temperatures in the interval 4.2-300 K. The magnetic field was created with the help of a bitter magnet with an induction of up to 14 $\mathrm{T}$ and a time of sweep over the field 1,75 T/min and 3,5 $\mathrm{T} / \mathrm{min}$ respectively for the temperature of the liquefied helium and in the temperature range 4.2-77 K. The fixed temperature of the cryostat insert is set using a special cryostat temperature regulation system. This system provides a gradual cooling of the cryostat to precisely set fixed temperatures. As a thermosensitive element of the thermocouple was used thermistor. In this case, the cryostat provides stabilization of the temperature is not worse $0.5^{\circ} \mathrm{C}$ during $20 \mathrm{~min}$.

All voltages are measured using digital voltmeters type Keithley-199 with accuracy is not worse $0.01 \%$. The current through the resistive branch is set from the current source Keithley-224 with accuracy $0.1 \%$, which determines the measurement error of the resistance.

\section{Experimental research results}

The basis of the work of semiconductor sensors of various physical quantities, in particular thermal and mechanical, is the temperature dependence of the electrical conductivity of the crystals and its change under the influence of external factors, for example deformations. When it comes to the creation of sensors for the low temperature interval, it should be borne in mind that in this case the electrophysical properties of the semiconductors will be determined by the degree of doping and the type of doping impurity, the degree of compensation and the degree of approach to the MIT. This necessitates the study of the effect of the degree of doping and the temperature behavior of the doping admixture on the electrical conductivity of semiconductor silicon crystals.

To establish mechanisms of low-temperature conductivity for the action of a magnetic field for microcrystals of p-type conductivity with a concentration of boron impurity corresponding to both the metal and dielectric side of MIT, the temperature dependences of resistance and magnetoresistance in the temperature range from 4,2 to $60 \mathrm{~K}$ in magnetic fields $(0 \div 14)$ Тл. Investigation of the temperature dependence of the resistance and magnetoresistance of silicon microcrystals with the concentration of the boron corresponding to the MIT from the dielectric side $\left(\rho_{300 \mathrm{~K}}=0.025 \Omega \times \mathrm{cm}\right)$ shown in Fig. 1,2.

As can be seen from Fig. 1, the nature of the resistance change in the temperature region $20-80 \mathrm{~K}$ в in general, the same, but in the low-temperature range 4.2$10 \mathrm{~K}$ in the slightly doped microcrystals there is a more sharp change in resistance. Significant dependence of crystal resistance with Fig. 1 is observed only to the temperature $10 \mathrm{~K}$, which is typical for the dielectric type of conductivity. With a decrease in the concentration of boron in silicon samples, that is, at a distance from the MIT in the dielectric region, the sensitivity of the crystals to the temperature increases, which manifests it self in increasing the resistance values. It can be used to design high-sensitivity temperature sensors with a thermoresistive principle of operation, or high-sensitivity 


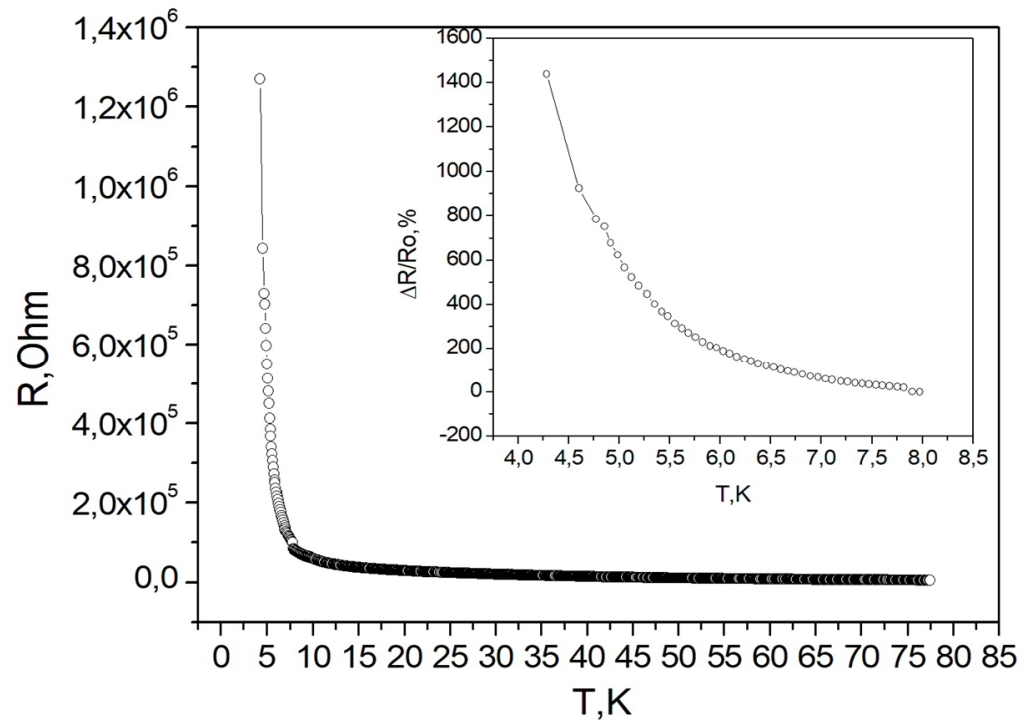

Fig. 1. Temperature dependence of the resistance of microcrystals $\operatorname{Si}\left(\rho_{300 K}=0.025 \Omega \times \mathrm{cm}\right)$ mixed with boron dopant concentration that meets the MIT of dielectric side and modified by nickel. Inset: relative change in the resistance of microcrystals in a narrow range of temperatures $4.2-9 \mathrm{~K}$.

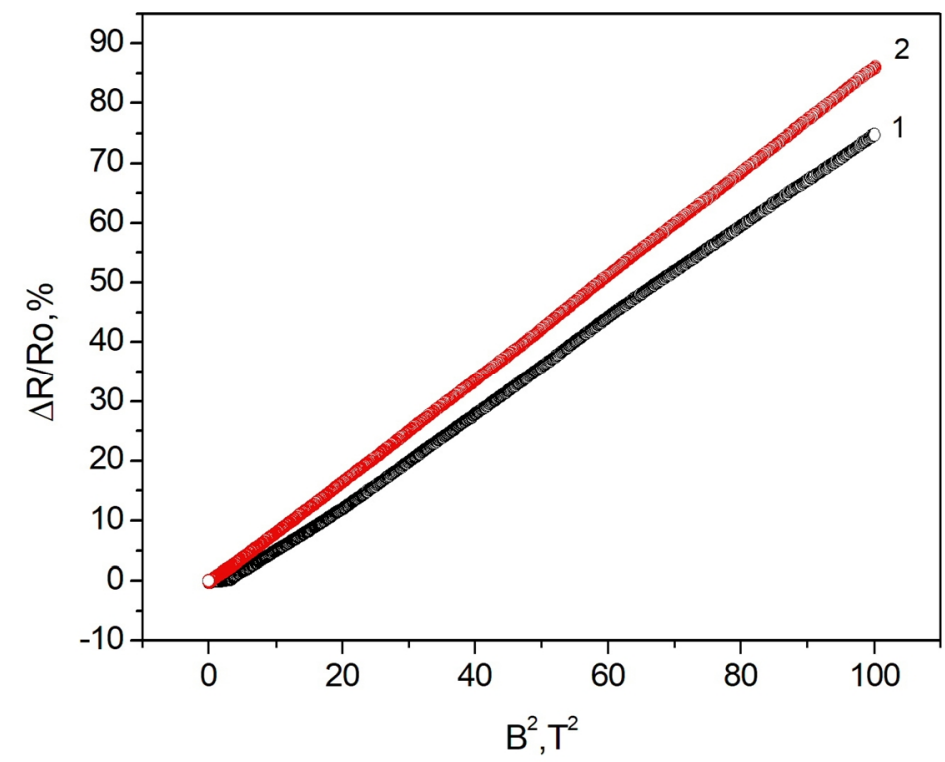

Fig. 2. Dependence of the magnetoresistance of microcrystals $\operatorname{Si}\left(\rho_{300 K}=0.025 \Omega \times \mathrm{cm}\right.$ ) from the induction of a magnetic field with an admixture of nickel and boron with a concentration of a doping admixture corresponding to the MIT from the dielectric side at a temperature 4.2K: 1- in longitudinal and 2- in transverse directions in relation to the direction of the magnetic field.

thermoreset. As can be seen from the insert to Fig. 1, the relative change in the resistance of the crystals varies by several orders of magnitude in the narrow range of temperatures. Temperature coefficient of resistance (TCR) such samples reach $300 \% \times K^{-1}$. Magnetoresistance behavior (Fig.2.) microcrystals $\mathrm{Si}<\mathrm{B}, \mathrm{Ni}\rangle$ at low temperatures corresponds to the classical pratolabolic change as for bulk silicon. It should also be noted that the deformation effect is observed only in Si p-type crystals with the concentration of the impurity corresponding to the approach to the critical transition concentration of the metal-dielectric and is absent in the Si p-type from the metal side of the transition, that is, on free holes. On the other hand, it decreases for microcrystals $\mathrm{Si} 3$ the concentration of boron corresponding to the dielectric side of the MIT and the deep dielectric region, that is, weakens in the case of transition to crystals with a reduced ensemble of localized holes [18]. The first factor indicates that for the effect of deformation of the charge carriers must be localized. The second factor indicates the need for sufficient concentration of localized magnetic dipoles, that is, possible some limiting interaction. In this case, each localized hole can be assigned a magnetic dipole moment that interacts with surrounding charge carriers and an external magnetic field. These effects were clearly observed during the study of silicon microcrystals with a concentration of a dopant boron in the vicinity of the transition metaldielectric $\left(N_{\mathrm{B}} \approx 5 \cdot 10^{18} \mathrm{~cm}^{-3}\right)$, which corresponds to the proximity of the MITs from the dielectric side.

Results of experimental studies of microcrystals $\mathrm{Si}<\mathrm{B}, \mathrm{Ni}>$ with a concentration of alloying admixtures of boron $N_{B}=5 \times 10^{18}$ at low temperatures is shown in Fig. 3,4. Evidently (Fig.3), that the presence of a magnetic 
impurity substantially affects the localized holes, in which the jump conductivity is realized, its ability to polarize the surrounding charge carriers. The monitored effect, which manifests itself at low temperatures, indicates the influence of the metal impurity on the transport of charge carriers at temperatures below $100 \mathrm{~K}$. Since at low temperatures there is a significant freeze of charge carriers, one of the dominant conduction mechanisms is the hopping conductivity, which depends directly on the concentration of impurities in the crystal. Thus, in Fig. 3 depicts the temperature dependence of the resistance $\mathrm{Si}<\mathrm{B}, \mathrm{Ni}>$ microcrystals for samples with boron concentration $N_{B}=5 \times 10^{18} \mathrm{~cm}^{-3}$, which corresponds to the proximity of the MITs from the dielectric side. As can be seen from Fig. 3, the presence of nickel affected the temperature dependence of the

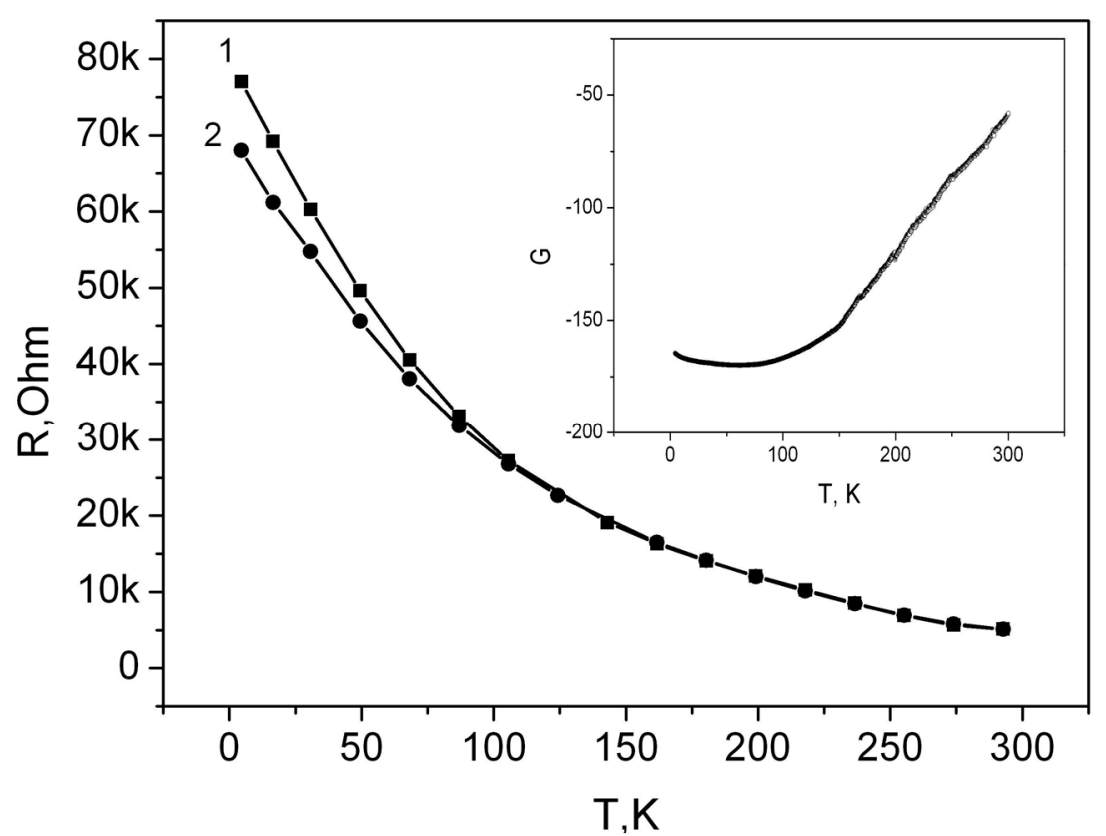

Fig. 3. Dependence of the resistance on the temperature of the crystals $\mathrm{Si}\langle\mathrm{B}\rangle$ for samples with boron concentration $\mathrm{N}_{\mathrm{B}}=5 \times 10^{18} \mathrm{~cm}^{-3}$, which corresponds to the proximity of the MIT from the dielectric side. Curves 1 and 2 correspond to the results of samples with an admixture of nickel, and without a magnetic impurity, respectively. On the insert: temperature dependence of the coefficient of strain sensitivity of microcrystals.

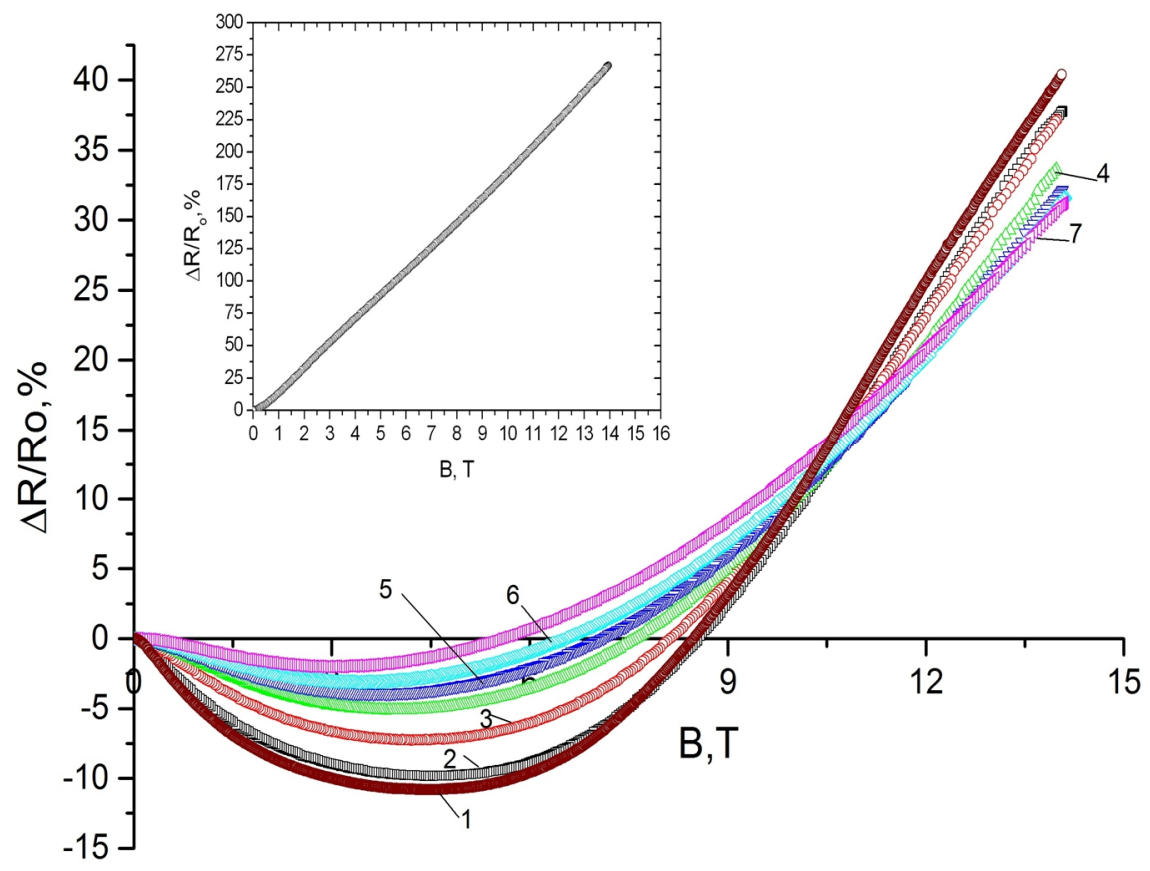

Fig. 4. Dependence of the magnetoresistance of microcrystals $\mathrm{Si}<\mathrm{B}, \mathrm{Ni}>\left(\rho_{300 \mathrm{~K}}=0.012 \Omega \times \mathrm{cm}\right)$ from induction of a magnetic field with a concentration of a doping boron impurity $\mathrm{N}_{\mathrm{B}}=5 \times 10^{18} \mathrm{~cm}^{-3}$, which corresponds to the proximity of the PMDs from the dielectric side for different temperatures: 1 - 4.2 K, 2 - $13 \mathrm{~K}, 3$ - $29 \mathrm{~K}, 4$ - $40 \mathrm{~K}, 5$ $50 \mathrm{~K}, 6-60 \mathrm{~K}$. On the insert: dependence of the magnetoresistance of silicon microcrystals on the induction of a magnetic field with the concentration of the boron $\mathrm{N}_{\mathrm{B}}=5,5 \times 10^{18} \mathrm{~cm}^{-3}$, which directly corresponds to the MIT. 


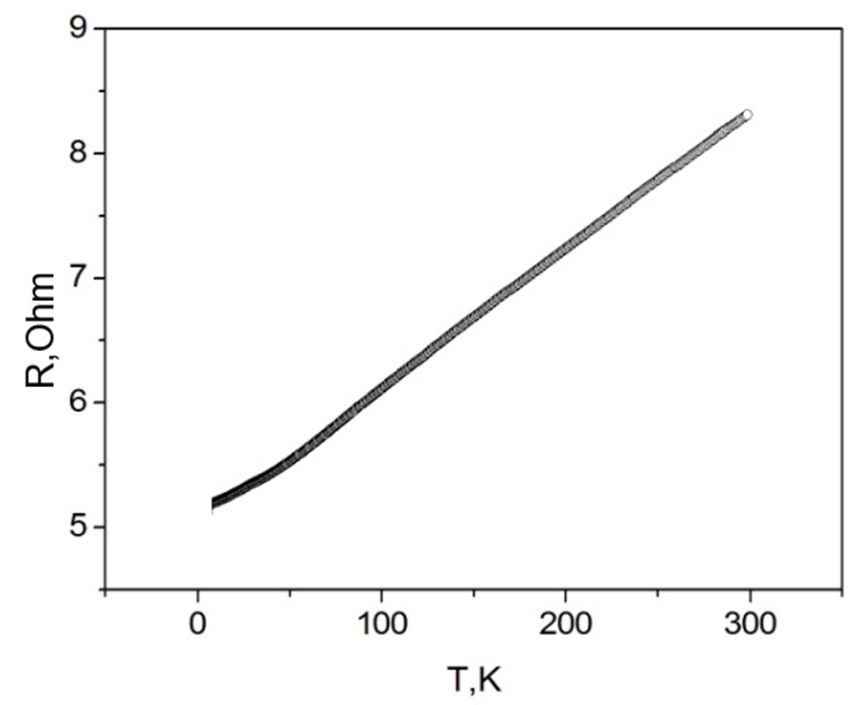

Fig. 5. Temperature dependence of the resistance of silicon microcrystals $\left(\rho_{300 \kappa}=0.007 \Omega \times \mathrm{cm}\right)$ with an admixture of nickel and boron with a concentration of a doping admixture corresponding to a metallic side to the MIT.

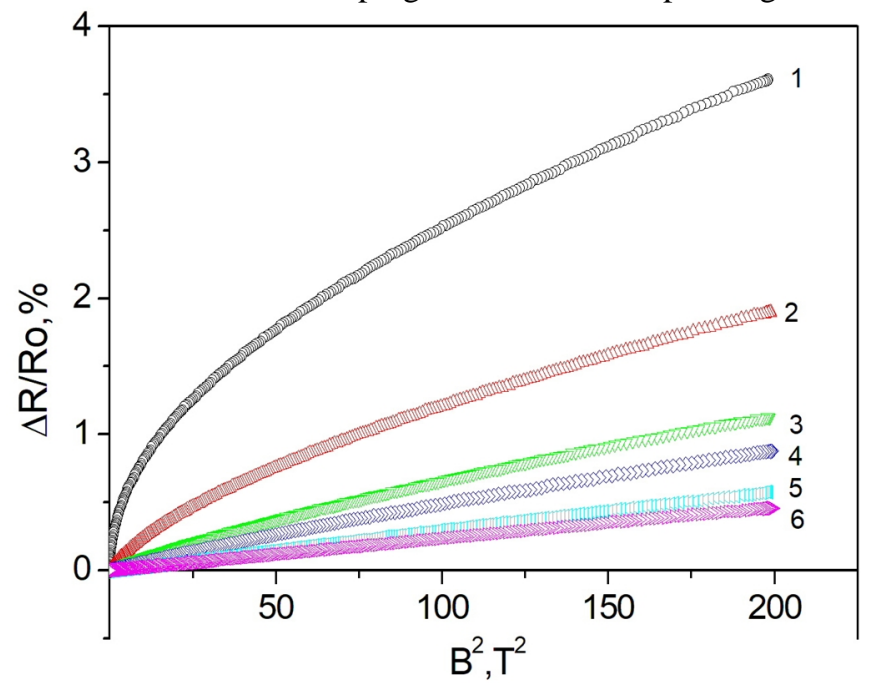

Fig. 6. Field dependence of the magnetoresistanse of microcrystals $\operatorname{Si}\left(\rho_{300 K}=0.007 \Omega \times \mathrm{cm}\right)$ with an admixture of nickel and boron with a concentration of alloying of boron, corresponding to the MIT from the metal side for different temperatures: $1-4.2 \mathrm{~K}, 2-13 \mathrm{~K}, 3-29 \mathrm{~K}, 4-40 \mathrm{~K}, 5-50 \mathrm{~K}, 6-60 \mathrm{~K}$.

microcrystal resistance, namely, an increase in the resistance value was observed, compared with crystals without a metal admixture. The localization of the atoms of nickel, as a free impurity, or the placement of a magnetic atom, as a defect in the crystal lattice of silicon (substitution), influenced the motion of the carriers in the crystal and led to their reduction, forming potential wells, or certain traps for carriers, resulting in a change in the crystal resistor. Experimental studies have shown that the conductivity of low-temperature silicon microcrystals is subject to the Mott's law $\left(\ln \rho \sim \mathrm{T}^{-1 / 4}\right)$. This confirms the dominance of low-temperature hopping conductivity $[12,15]$ as $R=R_{o} \exp \left(T_{o} / T\right)^{1 / n}$, were $n=1 / 3$ at $T<10 \mathrm{~K}$; $n=1 / 4$ at $T=10-25 \mathrm{~K} ; T_{o}-$ Mott temperature. In our studies, as in theoretical representations $[9,12,19]$ it is shown that the mechanism of conduction change satisfies the following correlation: $\rho \propto \exp E_{h o p} / k T$, were $E_{\text {hop }}-$ the activation energy of hopping conductivity by localized impurities, $T$ - temperature of observation [19].

Instead, due to the introduction of a magnetic impurity, the gap of the existence of a negative magnetoresistance has essentially expanded [15]. Transfer of holes (in a ferromagnetic mode) occurs with the help of the nearest neighbor by leap charge carrier on unoccupied places of localization. This assumes that the average distance between localized holes should be greater than the hole localization radius, i.e. $\alpha_{0}^{3} p<<1$, were $\alpha_{0}{ }^{3}$ represents the characteristic of the exponential recession of a hole wave function in localized states, $p$ effective concentration of holes [13]. We can assume that the interval of the existence of the hopping conductivity of the twice occupied states, which we previously announced in the interval of the existence of a negative magnetoresistance, has significantly increased.

Moreover, a negative magnetoresistance is usually observed in specimens with a concentration of impurity close to the critical concentration of MIT. The detected negative magnetoresistance in the microcrystals from the dielectric side of the MIT (Fig. 4) allows us to assume that, as in [20], in such samples, the appearance of a negative magnetoresistance can be caused by an increase 


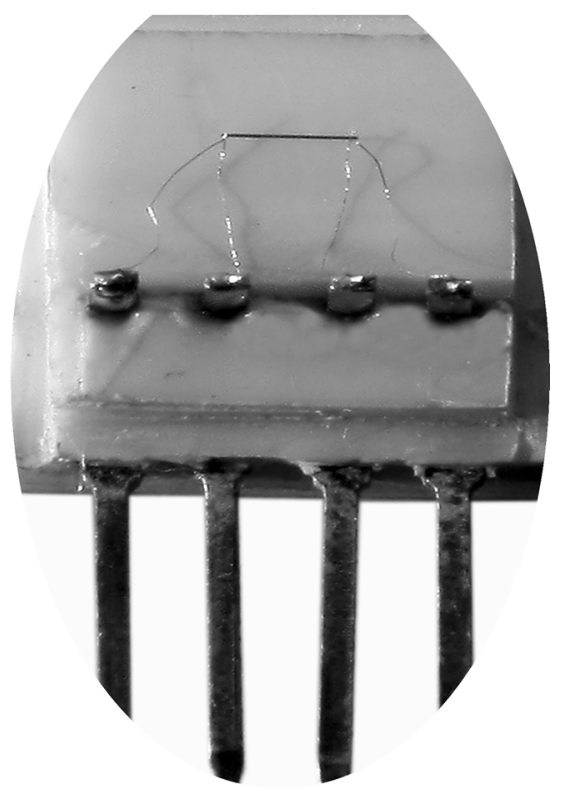

Fig. 7. General view of a magnetic field sensor with magnetoresistive principle of action.

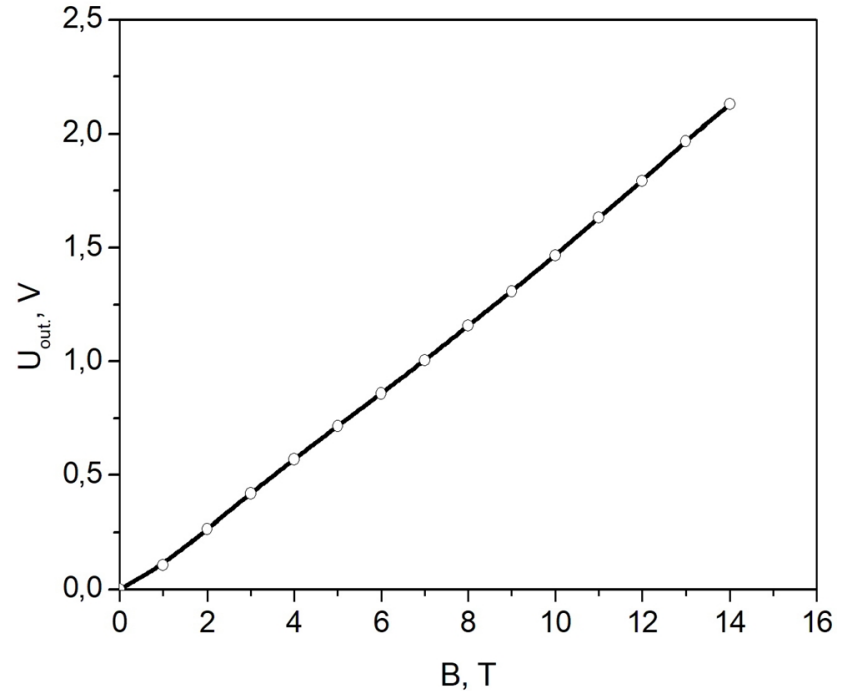

Fig. 8. Output signal of a magnetic field sensor with a magnetoresistive principle of action.

Table 1

Grading characteristic of the magnetic field sensor on the basis of doped boron and nickel-modified silicon microcrystals

\begin{tabular}{|l|l|l|l|l|l|l|l|l|}
\hline B, T & 0 & 2 & 4 & 6 & 8 & 10 & 12 & 14 \\
\hline $\mathrm{U}_{\text {out. }}, \mathrm{V}$ & 0 & 0.26 & 0.56 & 0.85 & 1.15 & 1.46 & 1.79 & 2.12 \\
\hline
\end{tabular}

\begin{tabular}{|l|c|c|c|c|c|c|c|c|}
\hline $\mathrm{B}, \mathrm{T}$ & 0 & 2 & 4 & 6 & 8 & 10 & 12 & 14 \\
\hline$\delta, \%$ & 1.9 & 0.04 & 0.06 & -0.67 & -1.03 & -0.84 & 0.24 & 1.61 \\
\hline
\end{tabular}

$\delta$ - relative error

in the conductivity of the hole pairs formed due to the ferromagnetic interacting of the delocalized carriers in the process of hopping conductivity. A characteristic feature of the negative magnetoresistance effect of the studied samples as in [21], which investigated the magnetoresistance heavily doped Ge $n$-type, there is a deviation from the quadratic dependence of the magnetoresistance on the magnetic field in the region of relatively weak fields (Fig. 4). The authors [21] explained the effect of the appearance of a negative magnetoresistance by the formation of " twain" - two states with paired spins, relatively close to each other and remote from the others, which exist near the Fermi level. Inside this "twain" obvious emergence of strong ferromagnetic interaction, which leads to the pairing of spins and thus to increase the conductivity of crystals. The effect of a negative magnetoresistance is associated with a change in the density of states near the Fermi level with a magnetic field. For a single ionization of "twain", its level for increasing the magnetic field shifts upwards. Part of the levels shifts downward, and the part upwards, which leads not only to the displacement of the Fermi level, but also to the change in the density of states in its vicinity.

In view of the use of detected effects in microcrystals $\mathrm{Si}<\mathrm{B}, \mathrm{Ni}>$ with the concentration of boron impurity, which corresponds to the closeness of the MIT during the development of sensors of physical quantities, it was found that the strain sensitivity coefficient for such samples in the region of helium temperatures reaches the value $\mathrm{K}_{4.2 \mathrm{~K}}=-165$ at deformation of compression $\varepsilon=-$ $5.29 \times 10^{-3}$ rel. un. (Fig. 3, inset). Instead, for the development of magnetic field sensors with a magnetoresistive principle of action, which is based on a significant magnetoresistive effect and reaches up to $250 \%$ (Fig.4, inset) microcrystals should be used $\mathrm{Si}<\mathrm{B}, \mathrm{Ni}>$ with a concentration of boron impurity, which corresponds directly to the MIT $\left(N_{B}=5,5 \times 10^{18} \mathrm{~cm}^{-3}\right)$.

For heavily doped silicon microcrystals (Fig.5,6) in all investigated temperature interval the typical metallic course of the temperature dependence of the resistivity characteristic, both for undeformed and for deformed crystals. Significant influence of deformation on characteristics of microcrystals was not observed. The weak dependence of the magnetoresistance Si with the metallic conductivity, due to the weak localization of the charge carriers, will be of fundamental importance in the application of such microcrystals as sensitive elements of sensors of thermal quantities, capable of operating in harsh conditions, in particular in high magnetic fields. At temperatures of liquefied helium, the maximum magnitude resistance reaches no more than $4 \%$ in magnetic fields with induction up to $14 \mathrm{~T}$. 


\section{III.Application}

The development of new branches of science and technology at the present stage (rocket space and aviation technology, cryogenic technology, cryoenergetics, etc.) puts forward the problem of creating miniature high sensors of mechanical, thermal, magnetic values, capable of working at low temperatures. Let's consider the solution of this problem on the example of creating sensors of magnetic quantities. At present, considerable attention is paid to heterostructures consisting of alternating magnetic (semimagnetic) and non-magnetic materials. For the application of an external magnetic field, electrons in this region are polarized and injections into a nonmagnetic region occur. As a result, a spinpolarized channel for transporting charge carriers appears in a nonmagnetic layer. The dependence of the magnetoresistance of such a sensitive element is not linear, and the magnetic saturation of the structure occurs in the magnetic field of 3-4 T, after which the sensor has a weak magnetic dependence.

The use of $\mathrm{Si}<\mathrm{B}, \mathrm{Ni}>$ microcrystals allows the creation of high-precision sensitive elements of a microelectronic sensor capable of operating under liquidhelium temperatures, provided that strong magnetic fields are applied up to $14 \mathrm{~T}$ with the ability to integrate sensitive elements with information processing schemes and low cost.

As a result of the research on the influence of the magnetic field, it has been established that the proposed sensitive element of the microelectronic sensor has a super-high sensitivity to the magnetic field (Fig. 4, inset), and the simplicity of the design provides a low inertia and at the same time high performance.

In fig. 7 shows a photograph of the general form of the sensor, and in Fig. 8 - the output characteristics of the sensor.

Grading characteristics of the magnetic field sensor with a magnetoresistive principle of operation is summarized in Table 1.

The sensor, based on the proposed sensing element, functions as follows. When the voltage is applied to the crystal contacts, an initial current will flow between them. Applying an external magnetic field effect on the polarization of the charge carriers in the surface layer of the crystal, resulting in a change in conductivity occur in the crystal. The efficiency of such a sensor is limited by the range of cryogenic temperatures (to the temperature of liquefied helium $4.2 \mathrm{~K}$ ) from the magnitude of the magnetic field to $14 \mathrm{~T}$ with a sensitivity of $5 \mathrm{mT}$.

\section{Conclusions}

As a result of studies of silicon microcrystals doped with boron transport impurities to concentrations corresponding to the MIT and nickel-modified, the features of electrophysical characteristics of specimens at low temperatures in strong magnetic fields up to $14 \mathrm{~T}$. It was found out that for the development of highsensitivity temperature sensors with a thermoresistive principle of action, or high-sensitivity thermoreset, it is necessary to use microcrystals $\mathrm{Si}<\mathrm{B}, \mathrm{Ni}\rangle$ with specific resistivity $\rho_{300 K}=0.025 \Omega \times \mathrm{cm}$, which corresponds to the dielectric region of the MIT. The relative change in the resistance of the crystals varies by several orders of magnitude at the temperatures of the liquefied helium $(4.2 \mathrm{~K})$. The temperature coefficient of resistance of such samples reaches $300 \% \times \mathrm{K}^{-1}$. For the development of sensors of mechanical quantities, it is suggested to use samples of silicon microcrystals with specific resistivity $\rho_{300 K}=0.012 \Omega \times \mathrm{cm}$. The coefficient of strainsensitivity for such samples in the region of helium temperatures reaches the value $\mathrm{G}_{4.2 \mathrm{~K}}=-165$ at deformation of compression $\varepsilon=-5.29 \times 10^{-3}$ rel. un. Weak dependence of the magnetoresistance Si with the specific resistance $\rho_{300 K}=0.007 \Omega \times \mathrm{cm}$ and the metallic nature of conductivity, due to the weak localization of charge carriers, will be of fundamental importance in the application of such microcrystals as sensitive elements of sensors of thermal quantities, able to operate in difficult operating conditions, in particular in high magnetic fields. At the temperatures of liquefied helium, the maximum magnitude resistance reaches no more $4 \%$ in magnetic fields with induction up to 14T. Instead, for the development of magnetic field sensors with a magnetically resistive principle of action, based on a significant magnetoresistive effect and reaching up to $250 \%$, it is necessary to use microcrystals $\mathrm{Si}\langle\mathrm{B}, \mathrm{Ni}\rangle$ with the concentration of boron impurity, which corresponds directly to the MIT $\left(N_{B}=5.5 \times 10^{18} \mathrm{~cm}^{-3}\right)$. The efficiency of such a sensor is realized in the range of cryogenic temperatures (to the temperature of liquefied helium $4,2 \mathrm{~K}$ ) from the magnitude of the magnetic field up to $14 \mathrm{~T}$ with a sensitivity of $5 \mathrm{mT}$.

Hoverko Yu.M. - doctor of technical sciences, senior researcher;

Shcherban N.O. - postgraduate.

[1]. A.A. Barlian, S.J. Park, V. Mukundan, B.L. Pruitt, Sens Actuators A, 134 (2007) 77-87.

[2]. A. Druzhinin, I. Maryamova, E. Lavitska, Y. Pankov, Sensors and Actuators: A. Physical, 68(1-3) (1998) 229233.

[3]. A. Fert, Thin Solid Films, 517, (2008) 2-5.

[4]. I. Zutic, J. Fabian, and S. Das Sarma, Rev. Mod. Phys., 76 (2004) 323.

[5]. D. Sanchez, C. Gould, G. Schmidt and L. W. Molenkamp, IEEE Trans. Electron Devices, 54 (2007) 984 - 990.

[6]. H. W. Wu, C. J. Tsai, and L. J. Chen, Appl.Phys. Let., 90 (2007) 043121.

[7]. E. Durgun, D. Cakir, N. Akman, and S. Ciraci, Phys. Rev. Lett., 99(25) (2007) 256806.

[8]. A. Kamra, B. Ghosh and T. K. Ghosh, J. Appl. Phys., 108 (2010) 054505.

[9]. A. Kaminski, S. Das Sarma, Phys. Rev. B, 68 (2003) 235210. 
[10]. A. Kaminski, S. Das Sarma, Phys. Rev. Lett., 88 (2002) 247202.

[11]. A. Druzhinin, I. Ostrovskii, Yu. Khoverko, S. Nichkalo, R. Koretskyy, Iu. Kogut, Phys. Status Solidi A, 211(2) (2014) 504-508.

[12]. S. Yatsukhnenko et al., Nanoscale Research Letters, 78(12), (2017) 1-7.

[13]. Liang Wei-Hua, Ding Xue-Cheng, Chu Li-Zhi, Deng Ze-Chao, Guo Jian-Xin, Wu Zhuan-Hua, Wang YingLong, Acta Phys. Sin., 59(11) (2010) 8071-8077.

[14]. A.A. Druzhinin, I. P. Ostrovskii, Yu. M. Khoverko, Iu. R. Kogut, S. I. Nichkalo, J. K. Warchulska, Journal of Nanoscience and Nanotechnology, 12 (2012) 8690-8693.

[15]. Anatoly Druzhinin, Igor Ostrovskii, Yuriy Khoverko, Sergij Yatsukhnenko, Journal of Nano Research, 39 (2016) 43-54.

[16]. A.A. Druzhinin, I. P. Ostrovskii, Yu. M. Khoverko, N. S. Liakh-Kaguj and Iu. R. Kogut, Materials Science in Semiconductor Processing, 14(1) (2011) 18-22.

[17]. A.A. Druzhinin, I. P. Ostrovskii, Yu. M. Khoverko, K. Rogacki et al, Journal of Magnetism and Magnetic Materials, 393 (2015) 310-315.

[18]. A. Druzhinin, I. Ostrovskii, Y. Khoverko, R. Koretskii, Materials Science in Semiconductor Processing, 40 (2015) 766-771.

[19]. S. Das Sarma, E. H. Hwang, A. Kaminski, Phys. Rev. B, 67 (2003) 155201.

[20]. Antonio Ferreira da Silva, Alexandre Levine and Zahra Sadre Momtaz, Henri Boudinovm, Bo E. Sernelius, Physical Review B, 91 (2015) 214414.

[21]. A.I. Veygner, A.G. Zabrodski, T.V. Tysnek, Semiconductors, 34(7) (2000) 774-782.

Ю.М. Ховерко, Н.О. Щербань

\section{Електропровідність та магнетоопір мікроструктур кремнію за низьких температур в околі переходу метал-діелектрик}

Національний університет «Львівська політехніка», м.Львів, вул Ст.Бандери, 12, 79013 khoverko@lp.edu.ua

Проведено комплексні дослідження мікрокристалів кремнію 3 питомим опором від $\rho_{300 к}=$

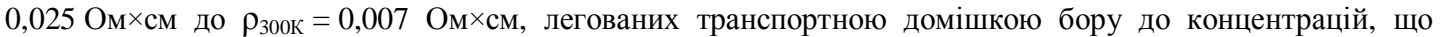
відповідають переходу метал-діелектрик та модифікованих домішкою перехідного металу нікелю за низьких температур до температури скрапленого гелію $\mathrm{T}=4,2 \mathrm{~K}$ в магнітних полях до 14 Тл. Визначено особливості електрофізичних характеристик зразків за низьких температур в сильних магнітних полях до 14 Тл, що обумовлені впливом магнітної домішки в розбавлених магнетитиками напівпровідниках i запропоновано викорастання таких кристалів в сенсорах фізичних величин (температура, магнітне поле, деформація).

Ключові слова: стрибкова провідність; мікрокристал; від’ємний магнітоопір; спін; кріогенні температури. 